\title{
Multipath Data Transfer Scheme for Virtual Private Networks
}

\author{
Mahalakshmi.C \\ Assistant professor \\ Department of Electrical Engineering \\ Annamalai University \\ Annamalai nagar
}

\author{
Ramaswamy. M \\ Professor \\ Department of Electrical Engineering \\ Annamalai University \\ Annamalai nagar
}

\begin{abstract}
The emerging automated world necessitates an elaborate infrastructure to comply with the growing traffic and ensure a reliable mode of communication. This paper proposes a multi path Virtual Private Network (VPN) with assuaging options through which remote sites are connected over a shared provider network. It articulates a scheme with a view to extract the best values for the performance metrics in the path that transfers the data with minimum utilization of bandwidth. The methodology encompasses measures to continue the process through the next minimum bandwidth path in the event of the occurrence of an exigency. It includes Network Simulator-2 (NS-2) results across a stream of flow to project its applicability in the context of creating a traffic free environment.
\end{abstract}

\section{Keywords}

VPN, Bandwidth, Multipath routing, MPLS.

\section{INTRODUCTION}

A Virtual Private Network (VPN) attempts to emulate the services offered by a private network and explores the feasibility to support the facilities of the service provider [1]. The incessant rise in traffic envisages enhanced provisioning to guarantee the desired bandwidth and an arrangement through which viable alternatives can be created. It is significant that the transfer mechanism enjoys a higher Quality of Service (QoS) and immunes it from extraneous considerations.

The recently evolving networks augur service guarantees among both the available models for a VPN [2]. The pipe model requires knowledge of the exact traffic demand between each VPN endpoint pair. On the contrary the hose model expects only to specify an aggregate ingress and egress bandwidth per VPN endpoint, which serves to reduce the complexities and make it more scalable. The hose model can thus be implemented over a variety of routing schemes and carries with it a host of advantages that includes flexibility, multiplexing gain and ease of characterization [3-5].

The advent of Multi-Protocol Label Switching (MPLS) has been found to address a host of shortcomings associated with Traffic Engineering and pave the way for improved network reliability, efficiency and service quality [6]. Two multipath constraint based routing algorithms using MPLS have been proposed for Internet Traffic Engineering. The simulation results have been found to enhance the success probability of path setup and utilization of network resources [7]. A modeling methodology has been developed to provide customers with flexible and convenient methods to specify the bandwidth needs of a VPN. The experimental results have been found to offer higher rejection ratio over other provisioning algorithms [8]. A new traffic splitting scheme has been outlined through an effective repartitioning approach. The performance has been compared with three other hybrid algorithms to exhibit its superiority [9]. The performance of multipath adaptive routing algorithms has been evaluated under different scenario like bottleneck link, traffic splitting among two output links of equal and unequal bandwidth paths. The results have been found to suggest a congestion reducing mechanism [10]. The hose model has been found to alleviate the scalability problem by reserving bandwidth for aggregate ingress and egress specifications in a VPN. The simulation study has been found to indicate the VPN trees constructed through the proposed approach to reduce the bandwidth requirements and enjoy a small execution time [11]. A multipath routing scheme has been suggested to improve the reliability and throughput of a Mobile Adhoc Network [12]. An algorithm has been proposed to spread traffic over alternate paths with a view to balance the load and increase the degree of fault tolerance [13].

The existing architecture may express its inability to enlarge its scope owing to economic compulsions. Besides, the bandwidth restriction echoes rituals and evinces a thirst for alternate paths of transmission. It is in this perspective that a methodology through which multi path data flow over progressive time frames can serve to arrive at the realistic need of the emerging scenario.

\section{PROBLEM DESCRIPTION}

The primary issues revolve around the optimum use of bandwidth and extradite a faultless transfer of data between customer sites. A strategy suitable for VPN that envisages provisioning using multi path mode is developed to handle contingencies and ensure continuity in the traffic. The strategy is realized through MPLS routing pattern in the NS-2 platform to project the minimum usage of bandwidth and derive the best performance in terms of its indices.

\section{SYSTEM MODELING}

The VPN customers extract the data through a hose architecture that specifies the incoming and outgoing traffic volume of each VPN endpoint (known as ingress bandwidth and egress bandwidth). While the ingress bandwidth of an endpoint is the capacity required for aggregating the incoming traffic to the endpoint from other endpoints, the egress bandwidth relates to that for the outgoing traffic. The hose model is scalable and allows the customer to manage the load on a per flow basis at the network edge, enabling the VPN provider to handle the flow inside the network [14].

A VPN pictorially visualized through Fig. 1 is constituted of a network where the nodes avail the facility to communicate with each other using a service provider. The state of the endto-end connection contributes to the reliability of the links and nodes, necessitating a seamless recovery to ensure service quality and availability in a VPN. The approach may involve 
circuitous routes to reroute the flow between the source and destination pairs affected by a link failure.

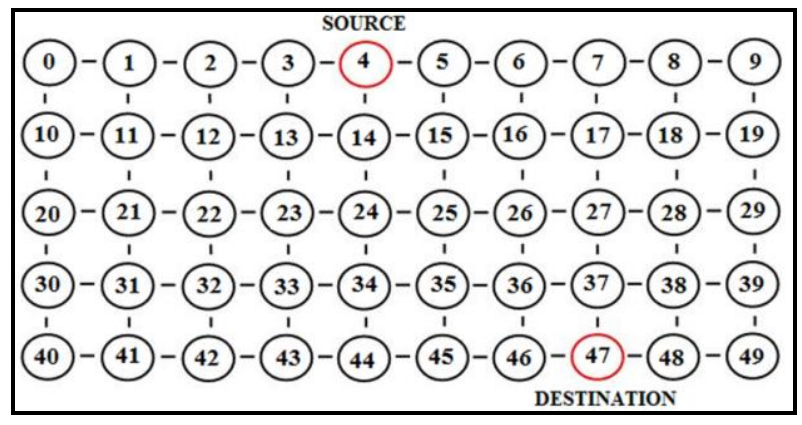

Fig 1: VPN Topology

\section{PROPOSED SCHEME}

A mechanism that establishes multiplicity of paths for traffic between a single source and destination invites attention in order to increase reliability of data transmission or to provide load balancing [15]. The algorithm uses a primary route to transmit data and it is only when the primary route fails, the scheme envisages the use of an alternative route. It is oriented to increase the overall network utilization, reduce the delay and packet loss properties and enjoy a higher transfer of data through the network.

The MPLS protocol traverses from one router to the next, with each router creating an independent forwarding decision for that packet. The packet headers contain the necessary information that is required to choose the next hop and choosing the next hop can therefore be thought of as the composition of two functions. While the first function partitions the entire set of possible packets into a set of Forwarding Equivalence Classes (FECs), the second maps each FEC to the next hop. The different packets of a particular FEC which travel from a particular node are likely to follow the same path or a set of paths associated with the FEC.

The assignment of a particular packet to a particular FEC is done just once, as the packet enters the network using the ingress router [16]. The FEC to which the packet assigned is encoded as a short fixed length value known as a "label". When a packet is forwarded to its next hop, the packets are "labeled" before they are forwarded. At subsequent hops the label is used as an index into a table which specifies the next hop and a new label. The old label is replaced with the new label, and the packet is forwarded to its next hop.

The MPLS routing continues to find interest in view of the fact that it supports explicit routing and provides basic mechanisms to facilitate secure traffic [7]. It allows a particular packet stream to follow a pre-determined path rather than a path computed by hop-by-hop destination based routing. However, this approach generally increases the overhead in the packet to be prohibitively expensive. With multiple Label Switched Paths (LSPs) available for an egress node, the goal is to distribute the traffic across the LSPs so that the network utilization as well as the network performance perceived by users is enhanced [17]. The flow chart seen in Fig. 2 explains the philosophy that is designed to support minimum usage of bandwidth and favour a wide range of QoS criteria.

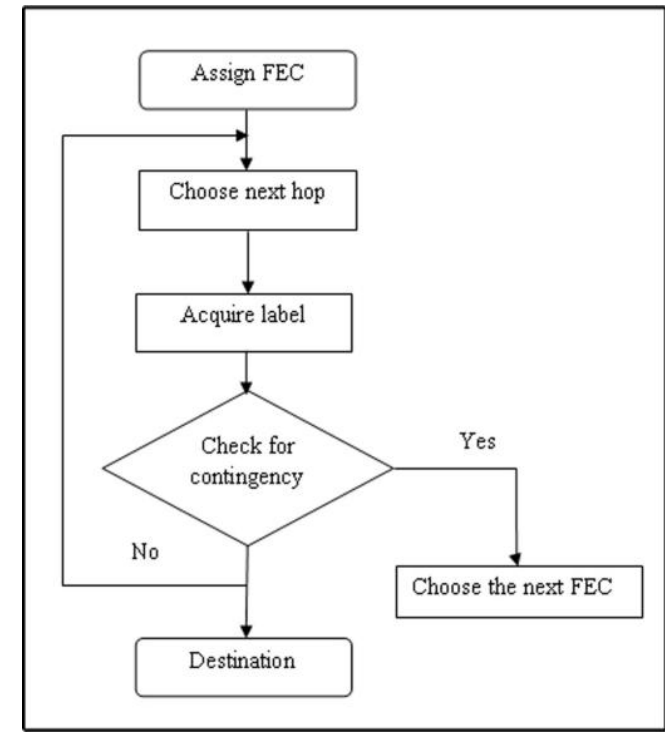

Fig 2: Proposed Flow Diagram

\section{SIMULATION RESULTS}

The methodology is implemented to evoke transfer of data through three paths one at a time over different frames of time span. It is perceived to espouse the second path in light of the creation of a contingency and thereafter the third. The NS-2 figures explaining the flow of data through the three paths is depicted in Figs. 3 to 5.

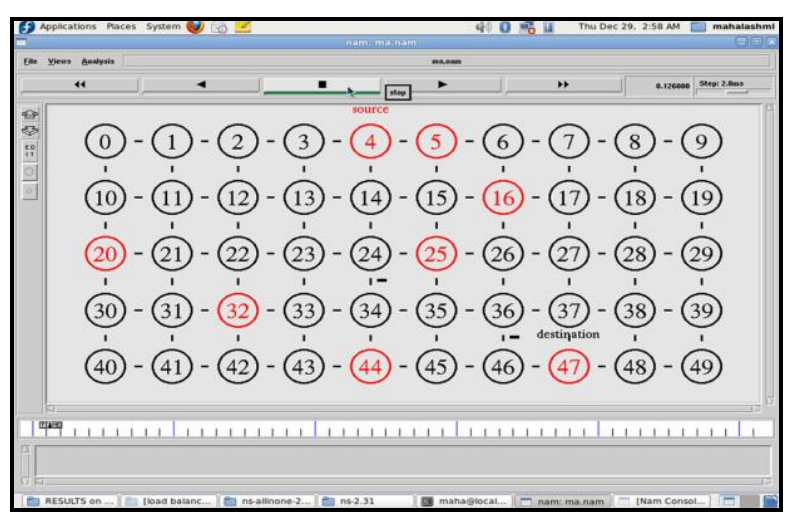

Fig 3: Data Flow in Path 1

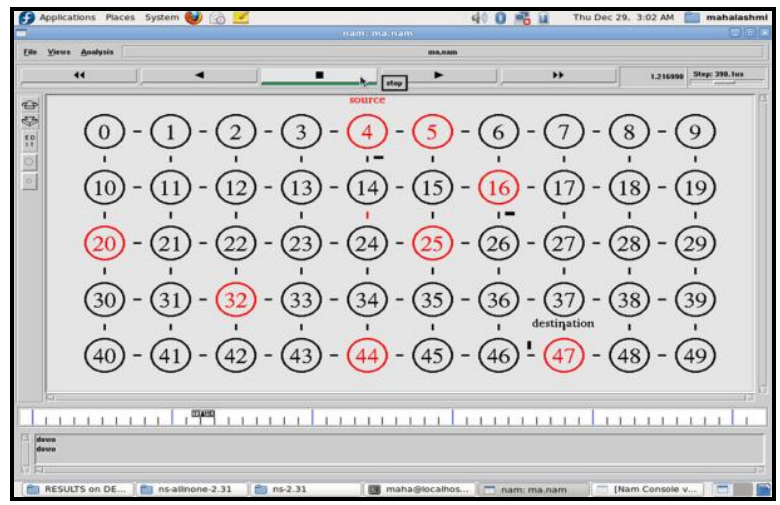

Fig 4: Data Flow in Path 2 


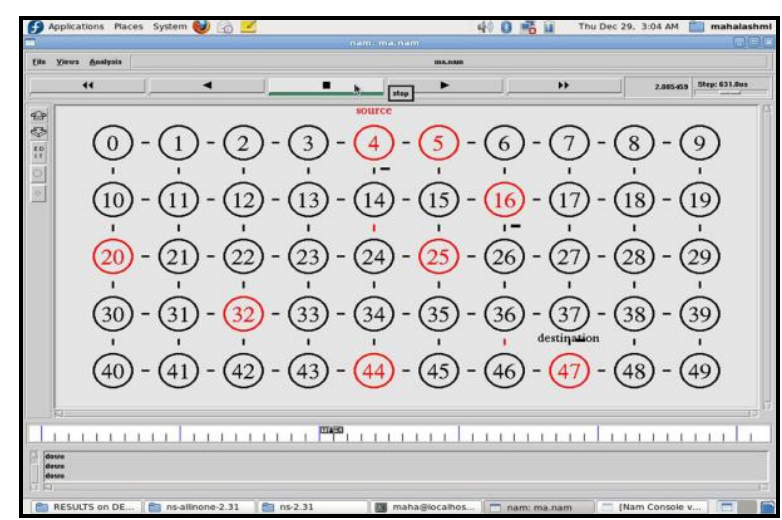

Fig 5: Data Flow in Path 3

The performance is evaluated using simulation for a data size of 1000 bytes in terms of packets received, routing delay, packet loss, energy expended and energy*delay metric for the three paths over their respective time frames and the results tabulated in Table1.

Table 1. Performance Metrics

\begin{tabular}{|c|c|c|c|c|c|c|}
\hline 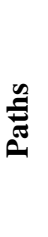 & 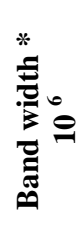 & 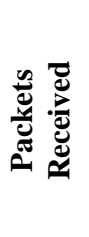 & 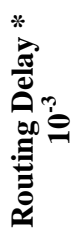 & 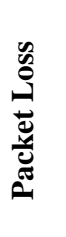 & 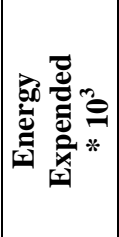 & 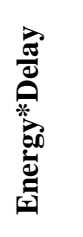 \\
\hline 1 & 0.48 & 368 & 13.8 & 184 & 10 & 138 \\
\hline 2 & 0.57 & 353 & 18 & 275 & 10.6 & 190.8 \\
\hline 3 & 0.62 & 283 & 34 & 370 & 12 & 408 \\
\hline
\end{tabular}

The NS-2 graphs displayed through Figs.6 to 10 relate to the indices of the minimum bandwidth path in its time span. It extricates the ability of the methodology to arrive at a linear increase in their values except for the delay which arbitrates a high start up time and there after reasons out a decline through the projected time frame.

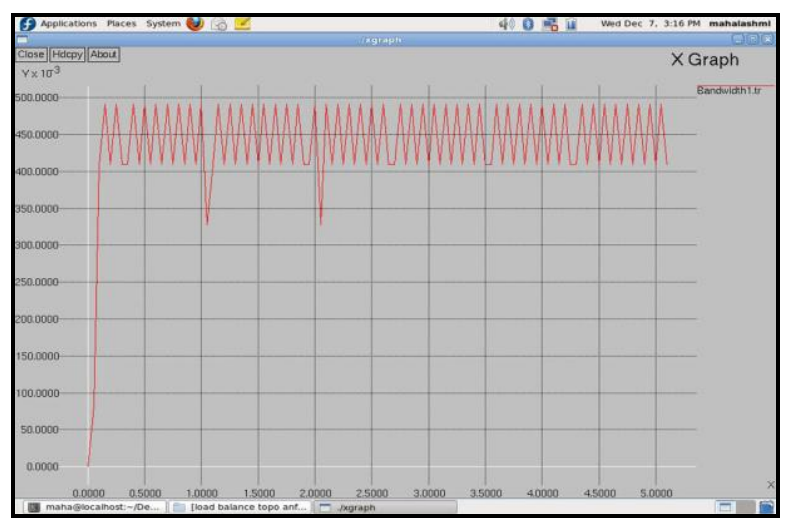

Fig 6: Bandwidth vs. Time

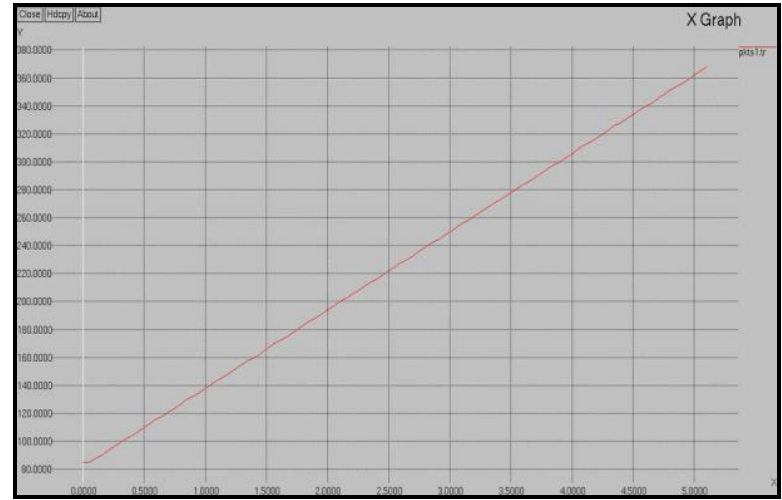

Fig 7: Packets Received vs. Time

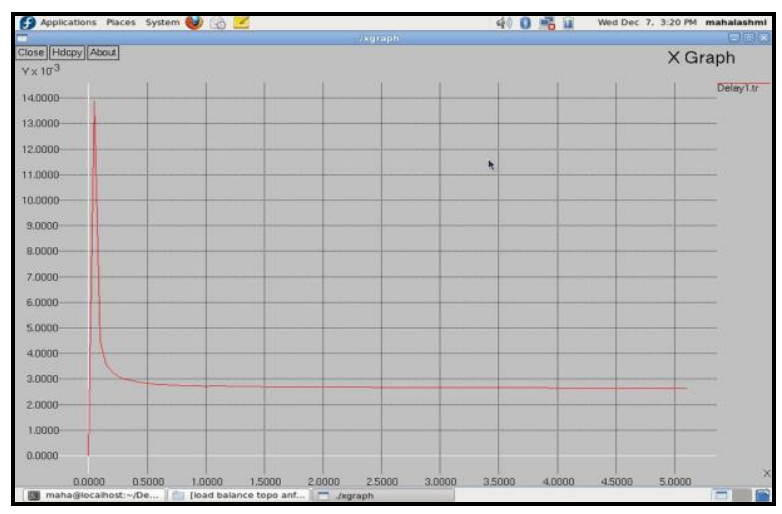

Fig 8: Routing Delay vs. Time

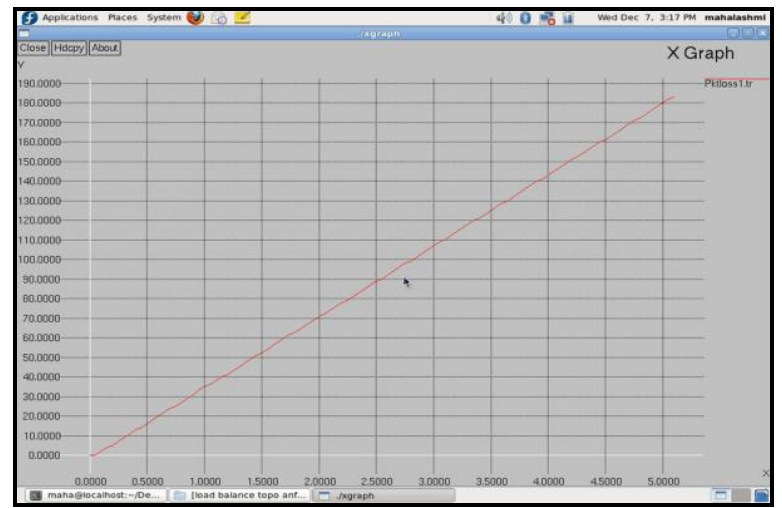

Fig 9: Packet Loss vs. Time

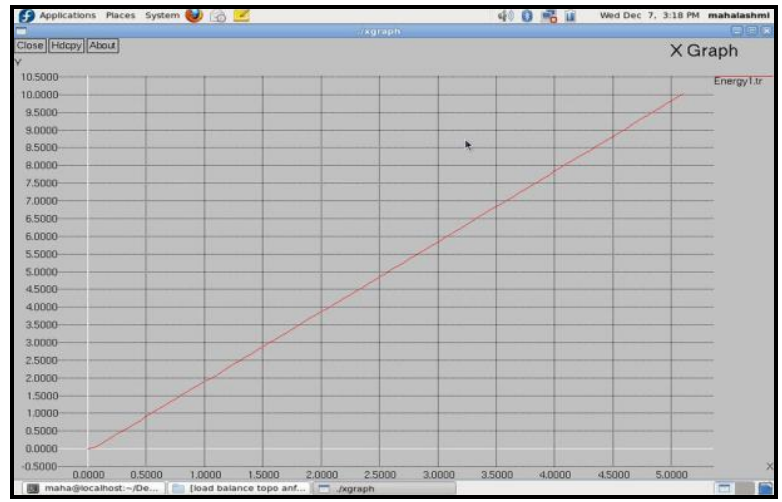

Fig 10: Energy vs. Time 
The size of the packets is allowed to increase and the performance investigated to explore the suitability of the algorithm for large scale transmission through the indices. The bar diagrams in Figs. 11 and 12 relate the increase in the number of packets received and associated fall in the transmission delay respectively over a viable operating range for the chosen architecture in the path which uses the minimum bandwidth, thus further highlighting the significance of the proposed routing methodology. However, there is an increase in the loss of packets and the energy expended as seen from Figs. 13 and 14. The network Packet Delivery Ratio (PDR) from Fig. 15 is observed to decline with increase in packet sizes due to the constraints in its load handling capability. The values of the different metrics project to realize the robustness of the scheme in the sense the minimum bandwidth path continues to enjoy the best results over a wide range of data size.

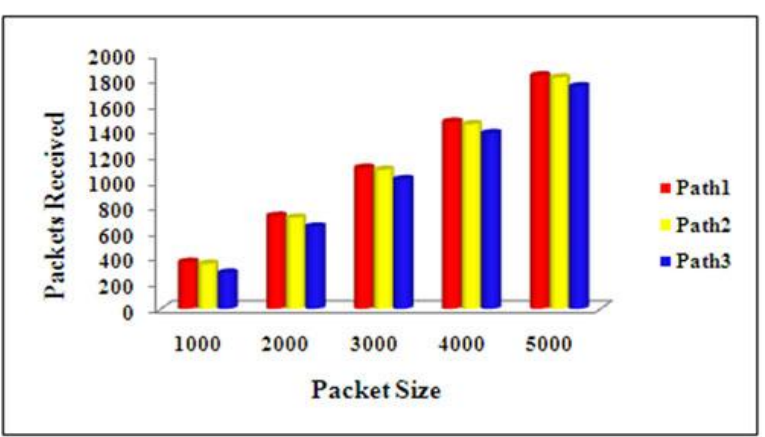

Fig 11: Packets Received vs. Packet Size

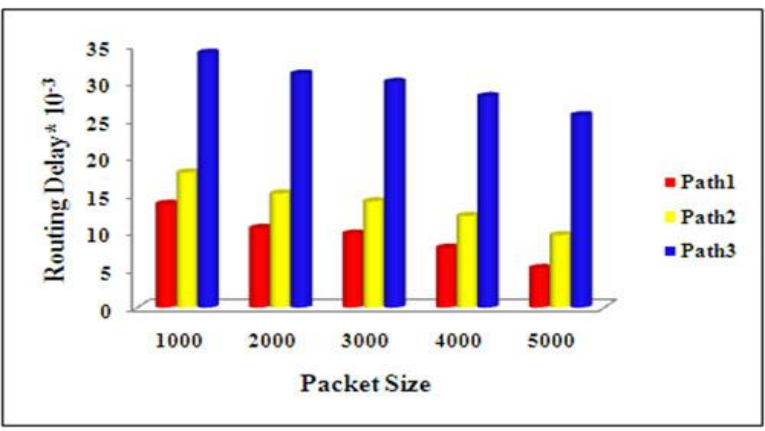

Fig 12: Routing Delay vs. Packet Size

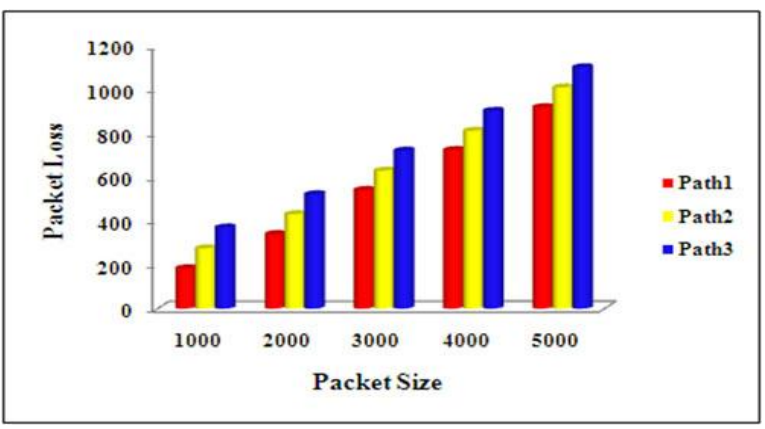

Fig 13: Packet Loss vs. Packet Size

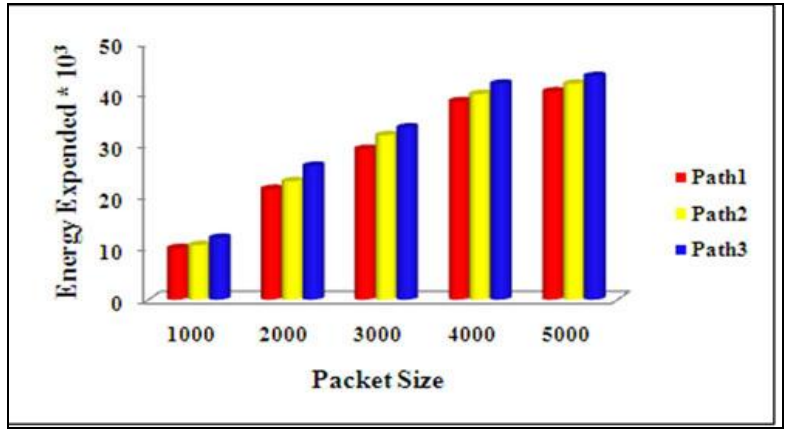

Fig 14: Energy Expended vs. Packet Size

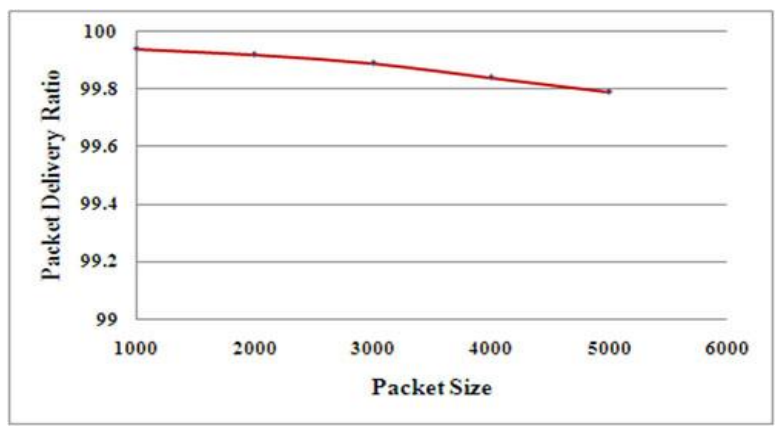

Fig 15: Packet Delivery Ratio vs. Packet Size

\section{CONCLUSION}

A Multi path routing scheme has been developed using the hose model of VPNs. The scheme has been tailored to enforce an effective mechanism to display the significance of minimum utilization of bandwidth in the evolving traffic scenario. The algorithm has been implemented using a chosen network model and its performance evaluated through simulation. The results have been found to reveal degrees of service in proportion to their bandwidths. It has been brought out that this routing technique effectively annihilates the potential contingency problems in the service provider network. It can be inferred that the availability of multiple paths between each VPN endpoint pair will prevail over link failures within the VPN through the innovation of new algorithms.

\section{ACKNOWLEDGMENTS}

The authors thank the authorities of Annamalai University for providing the necessary facilities in order to accomplish this piece of work.

\section{REFERENCES}

[1] Ravi. R, and Radhakrishnan. S, 2007. Enhanced restoration algorithm in Virtual Private Network with QoS Support. Asian journal of Information Technology. 6(10), 1035-1040.

[2] Yu-Liang Liu, Yeali. S, Sun, and Meng Chang Chen. 2005. Traffic Engineering for Hose-Model VPN Provisioning. IEEE Globecom. 2, 1080-1085.

[3] Giuseppe. F, Italiano, Rajeev Rastogi, B’ulent Yener. 2002. Restoration Algorithms for Virtual Private Networks in the Hose Model. Twenty-First Annual Joint Conference of the IEEE Computer and Communications Societies. 
[4] Duffield. N. G., Goyal. P., Greenberg .A., Mishra .P, Ramakrishnan .K.K, and Van Der Merwe .J.E. 2002. Resource Management with Hoses: Point-to- Cloud Services for Virtual Private Networks. IEEE/ACM Transactions on Networking. $679-692$.

[5] Gee-Swee Poo, and Haibo Wang. 2007. Multi-path routing versus tree routing for VPN bandwidth provisioning in the hose model. Computer Networks. 51(6), 1725-1743.

[6] Daniel .O, Awduche, Bijan Jabbari 2002. Internet traffic Engineering using Multi-Protocol Label Switching. Journal of Computer Networks. 40(1), 111-129.

[7] Ho Young Cho, Jae Yong Lee, and Byung Chul Kim. 2003. Multi-path Constraint-based Routing Algorithms for MPLS Traffic Engineering. IEEE International Conference on Communications. 1963-1967.

[8] Yu-Liang Liu, Yeali .S, Sun and Meng Chang Chen. 2005. On the On-Line Hose-Model VPN Provisioning. Advanced Industrial Conference on Telecommunications. 53-58.

[9] Kyeongja Lee, Armand Toguyeni, and Ahmed Rahmani. 2006. Hybrid multipath routing algorithms for load balancing in MPLS based IP network. International Conference on Advanced Information Networking and Applications. 1, 1-6.

[10] Yashpaul Singh .E.R., Soni. M. K. and Swarup .A. 2007. Simulation Study of Multi-Path Routing Algorithm in Different Situations. International Journal of Computer Science and Network Security, 7(11), 295297.
[11] Monia Ghobadi, Sudhakar Ganti and Gholamali C. Shoja. 2007. Hierarchical Provisioning Algorithm for Virtual Private Networks Using the Hose Model. Global Telecommunications Conference. 2467- 2471.

[12] Soundararajan.S, and Bhuvaneswaran R.S., 2011. Multipath Routing Backbone for Improving QoS in Mobile Ad hoc Networks. European Journal of Scientific Research, 53(2), 222-230.

[13] Pratheema.S., Srinivasagan.K.G., and Naskath. J. 2011 Minimizing End-to-End Delay using Multipath Routing in Wireless Sensor Networks. International Journal of Computer Applications, 21(5), 20-26.

[14] Tat Wing Chim, King-Shan Lui, Kwan L. Yeung and Chi Ping Wong. 2005. Routing Algorithm for Provisioning Symmetric Virtual Private Networks in the Hose Model. Global Telecommunications Conference. 802-806.

[15] Rosen .E, Viswanathan .A, and Callon .R. Multiprotocol Label Switching Architecture. RFC 3031, IETF, January 2001.

[16] Zenghua zhao, Yantai shu, Lianfang zhang, and Oliver yang. 2005. Flow-level multipath load balancing in MPLS network. IEICE Transaction on Communications. E88-B (5), 2015-2022.

[17] Koushik Kar, Murali Kodialam, and Lakshman .T.V. 2000. Minimum Interference Routing of Bandwidth Guaranteed Tunnels with MPLS Traffic Engineering Applications. IEEE Journal on Selected Areas in Communications.18(12),2566-279. 